\title{
Are spatial and temporal attention independent?
}

\author{
Alicia MacKay and James F. Juola \\ University of Kansas, Lawrence, Kansas
}

\begin{abstract}
Participants searched for one of two target letters in a rapid serial visual presentation (RSVP) sequence of 17 successive frames, each containing four letters arranged into a box around a central fixation point. In control trial blocks, the participants had no information about when or where one of the target letters would appear. In other trial blocks, visual cues were given to indicate with $100 \%$ validity either the spatial location of the target, the time at which it would be presented, or both where and when it would appear. The results showed that both types of cues were effective on their own in speeding target identification, and their effects combined additively when the cues were presented and used together. These results support a growing body of evidence indicating that early attentional selection of information in vision is independently attuned to spatial and temporal properties of the environment.
\end{abstract}

The typical environment provides much more sensory stimulation than can be processed effectively at any moment. Therefore, attention seems to have evolved as a means to select relevant and important information for deeper analysis while rejecting irrelevant information. Our knowledge of spatial relations and other aspects of physical reality leads us to expect certain objects in specific locations and in certain types of arrangements with other objects. Similarly our knowledge of cause and effect relations leads us to expect certain outcomes for specific events. Thus knowledge-based, endogenous deployment of visual attention over space and time should typically enhance our ability to recognize selected objects and anticipate expected events. The question addressed in the present research is whether spatial knowledge, that directs us to locate and identify objects, and temporal knowledge, that enables us to anticipate the outcomes of events, operate independently or cooperatively in selecting useful information from the environment.

Decades of research have shown that target identification time and accuracy are facilitated when attention is directed in advance to the target's location by a valid spatial cue, and that costs are found when such cues are invalid (e.g., Juola, Bouwhuis, Cooper, \& Warner, 1991; Nakayama \& Mackeben, 1989; Posner, 1980). Although there have been numerous studies investigating spatial attention, there have been relatively fewer studies of the allocation of attention over time. Research in diverse areas such as determining the optimal advance time for warning signals (e.g., Karlin, 1959), the length of time that observers can maintain a heightened state of vigilance (e.g., Mackworth, 1948), and the loss of information during an attentional blink (AB; Raymond, Shapiro, \& Arnell, 1992) has shown that attention can be directed effectively to temporal intervals of various lengths (see also Casini
\& Macar, 1997). By the same token, performance suffers when relevant information occurs outside the window of heightened attention. In research demonstrating voluntary control of attention over time, cues referring to specific temporal intervals can result in a decrease in response time (RT) when the temporal expectancy of an event matches the actual time of its occurrence (Correa, Lupiáñez, Milliken, \& Tudela, 2004; Griffin, Miniussi, \& Nobre, 2001). It is likely, therefore, that valid information about when a target will occur can be just as beneficial as knowledge about where a target will occur in terms of measured speed and accuracy of target identification.

There have been only a handful of studies making direct comparisons of the relative effectiveness of spatial and temporal cues in visual attention. Recently two methods have been used in which spatial and temporal attention have been investigated simultaneously. One method examines how temporal and spatial aspects of attention impact each other, such as by adding a spatial component to an $\mathrm{AB}$ task and investigating the distribution of effects that occur in spatiotemporal orienting (Kristjánsson \& Nakayama, 2002). The second method uses cues that have both spatial and temporal properties (Coull \& Nobre, 1998; Doherty, Rao, Mesulan, \& Nobre, 2005; Milliken, Lupiáñez, Roberts, \& Stevanovski, 2003). Both methods have shown that spatial and temporal information can be used simultaneously to improve attentional selection over that obtained by the use of either cue alone.

The purpose of the current research is to investigate whether or not spatial attention and temporal attention depend on the same underlying mechanisms. To date there have been inconsistent findings as to whether they do (e.g., Casini \& Macar, 1997; Doherty et al., 2005; Jiang \& Chun, 2001; Milliken et al., 2003). This question has been addressed through the combined use of spatial, temporal,

A. MacKay, anm1619@ku.edu 
and spatiotemporal cuing tasks. A control condition measures performance when attention presumably is diffusely distributed over a display for the length of a given trial. In contrast, in other conditions, attention should be focused narrowly over a specific location or to a specific moment of time, or to both a specific time and place if it is possible for the participant to do so. To the extent that such focus is effective, then information present at the cued location or within the cued time interval should be selected for further processing, whereas information with other spatial or temporal parameters will fail to be selected or even actively inhibited (Nakayama \& Mackeben, 1989).

Previous findings showing benefits from spatial cues (Posner, 1980) and temporal cues (Griffin, Miniussi, \& Nobre, 2001, 2002; Karlin, 1959) separately were replicated in the present study. In addition, the present experiment also replicated and extended the more recent findings of added benefits when spatial and temporal cues are provided together (Coull \& Nobre, 1998; Doherty et al., 2005; Milliken et al., 2003).

The main purpose of the present study was to extend previous findings by using more sensitive measures of spatial and temporal cue effectiveness. That is, our research used a target discrimination task, rather than simple detection, such as that used in the Coull and Nobre (1998) and Doherty et al. (2005) studies. In addition, the target appeared in one of four different locations, and in one of six possible lags, that were individually cued, rather than only a small number of possible spatial and temporal positions (e.g., two in each case in the Coull and Nobre study and three temporal lags in the Milliken et al. study). The addition of a greater range of spatial locations and temporal lags in the present study allows the cues to effect a greater reduction of uncertainty in both dimensions as opposed to earlier research, thus engendering stronger and more consistent cuing effects. The aim of the current study was to provide a quantitative test of the relative magnitudes and type of interaction found when spatial, temporal, or both types of information are provided to locate and identify a target in an RSVP stream. It is quite likely that orienting to positions in space for the purpose of identifying objects and orienting to moments in time for the purpose of interpreting events are based on separate neurological sub- strates, analogous to the "where" and "what:" substrates of the respective dorsal and ventral streams in higher visual processing (Mishkin, Ungerleider, \& Macko, 1983). If attention to objects in space and episodes in time are controlled by independent mechanisms, then we should find that their effects combine additively when the two types of cues are used in combination. The present results supported this conclusion.

\section{METHOD}

\section{Participants}

The participants were 18 members of the university community enrolled in or assisting with an advanced undergraduate psychology course at the University of Kansas. All participants had normal or corrected-to-normal vision.

\section{Apparatus and Stimuli}

E-Prime software (Schneider \& Zuccolotto, 2002) was used to control stimulus presentation and data collection. The experiment made use of a $2.4 \mathrm{GHz}$ Pentium 4 processor connected to a 19-in. VGA monitor. The letters " $R$ " and "L" were chosen as targets, and the letters $\{\mathrm{C} \mathrm{G} \mathrm{J} \mathrm{M} \mathrm{N} \mathrm{O} \mathrm{S} \mathrm{T} \mathrm{UV} \mathrm{WY} \mathrm{Z}\}$ were chosen as distractors with an aim to minimize confusability among target and distractor letters. The letters were shown in 32-point bold, Arial font in black against a white background. Each display consisted of a black central fixation cross and four letters positioned to form a box around the cross. The cross and the letters measured about $2 \mathrm{~cm}$ square, which, from a viewing distance of about $90 \mathrm{~cm}$ maintained by a chinrest, corresponded to a visual angle of about $1.3^{\circ}$ on a side. The corners of a larger box were indicated by four black isosceles triangles, with sides $2 \mathrm{~cm}$ in length, and these were placed about $6.5 \mathrm{~cm}$ from the center of the fixation cross which corresponds to about $4^{\circ}$ of visual angle (see Figure 1). For eight of the 18 participants an ASL model 504 eye-tracker with pan-tilt optics was used to track and record eyemovements at a rate of $120 \mathrm{~Hz}$ to ensure that eye movements were not made in response to the cues provided.

\section{Procedure}

The task was visual search for either of two targets using an RSVP sequence of 17 frames on each trial. The frames were shown for $117 \mathrm{msec}$ each, with a blank-screen interstimulus interval of $13 \mathrm{msec}$ (stimulus onset asynchrony $=130 \mathrm{msec}$ ). Each frame contained four different letters, and one target ( $\mathrm{R}$ or $\mathrm{L}$ ) appeared in one of the four quadrants on only one of the frames $(2-16)$ per trial. Distractor letters were randomly selected to fill the other quadrants and frames with the constraint that no letters were ever repeated within a frame or on two successive frames.

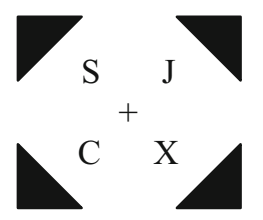

No-Cue Control

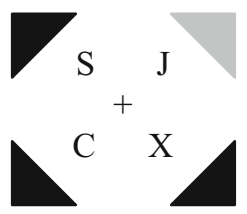

Spatial Cue

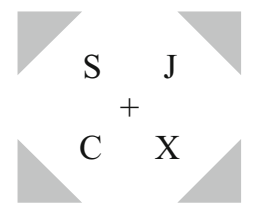

Temporal Cue

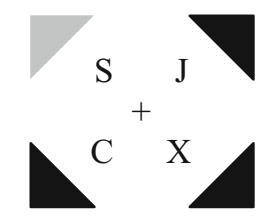

Spatiotemporal Cue

Figure 1. Schematic layout of display conditions and comparisons of the cues used in the three cuing conditions (approximately to scale). Gray is used to indicate which triangles would be red during the trial, and black is used to indicate which triangles would be black. In the control (no-cue) condition, black triangles were presented on every frame. In the spatial cue condition one of the corner triangles was red on the first frame. In the temporal cue condition, all four of the triangles were red on one of the later frames. In the spatiotemporal cue condition, one of the triangles was red on one of the later frames. In both the temporal and spatiotemporal cue conditions, the target followed the cue by a set number of frames $(0-5)$ that was held constant within a block of trials. 
Each subject first participated in a practice session in which no information about where or when the target would appear was given (control condition). The practice session consisted of a 20 -trial block followed by three blocks of 64 trials each. Over all trial blocks, the target R or L appeared at least two times in each combination of frame (2-16) and quadrant (upper left, upper right, lower left, lower right).

After the practice session, participants completed four additional experimental sessions on different days that included the control condition, spatial cue condition, temporal cue condition, and spatiotemporal cue condition. The orders in which these four sessions were carried out were counterbalanced across subjects by using a Latin square. In all conditions, the cues were $100 \%$ valid, and participants were instructed to maintain fixation on the central cross while attempting to use the cues. The participants were also given verbal and written instructions to use the spatial, temporal, and spatiotemporal cues, when relevant, before each block of trials. There were 20 practice trials and 3 experimental blocks of 64 trials each in the control and spatial cue conditions (192 trials per session).

In the spatial cue condition one of the four black triangles was shown in red on the first frame of the RSVP sequence. The red triangle was an explicit cue in that it cued the adjacent location where the target would appear later in the letter stream.

In the temporal cue condition, all four of the triangles appeared in red on one of the frames (Frame 2, 5, 8, or 11, randomly) in the RSVP sequence. The color change was a symbolic temporal cue that indicated when the letter was to appear. In separate blocks of trials, the target appeared either in the same frame as the red triangles or 1 , $2,3,4$, or 5 frames later (lags $0-5$ ). The orders in which participants went through blocks with specific cue-target lags were counterbalanced by using a Latin square.

In the spatiotemporal cue condition, one of the four black triangles appeared in red on one of the frames (again, Frame 2, 5, 8, or 11 , randomly) in the RSVP sequence. The color change indicated explicitly where the target would appear and implicitly when the target would appear. The target always appeared in the cued location, and in separate blocks of trials the target appeared either in the same frame as the red triangle, or 1,2,3, 4, or 5 frames later. Again, the orders of the trial blocks for any specific cue-target lag were determined by using a Latin square, and there were 20 practice trials and 56 experimental trials at each lag (336 experimental trials per session) for both the temporal and spatiotemporal conditions.

In all conditions the trial sequence was the same. Participants were to detect and identify the presence of a target letter, $\mathrm{R}$ or $\mathrm{L}$, as rapidly as possible while attempting to minimize errors. They were told that the cues were $100 \%$ valid and that they should try to use them to aid in target discrimination. In the temporal and spatiotemporal conditions, they were told before each block what specific temporal lag would occur between the cue and the target in that block. Each trial began with a $500 \mathrm{msec}$ exposure of the central fixation cross followed by the 17 RSVP frames. One of the targets appeared once, in Frames 2-16. As soon as the target was detected participants were to push the "Z" key on the keyboard if they detected the target letter L or the "?" key if they detected the target letter R. Feedback was provided when an error was made by the presentation of a 500-msec beep immediately after the error.

Even though participants were instructed not to move their eyes, and we believe that they seldom did, we could not be certain, and eye movements could confound the results in the spatial and spatiotemporal cue conditions. Therefore, we decided to measure eye movements during the search task for about half of the subjects. The procedure for the eight subjects whose eye fixations were monitored was identical with one exception. A second type of feedback was provided at the end of each trial in the form of a chime that sounded when the eye tracking system detected an eye movement of more than $1^{\circ}$ away from the central fixation cross (i.e., about halfway from the cross to one of the display positions) at any time during a trial. When an eye-movement was made, that trial was skipped and the program proceeded to the next trial. At the end of each block, those trials that had been skipped were then repeated until they were completed without eye movements.

\section{RESULTS}

Only trials in which the target was correctly identified were included in the RT analysis. Trials resulting in RTs greater than 2,000 msec or less than $200 \mathrm{msec}$ were also deleted. These restrictions led to the exclusion of fewer than $7 \%$ of the trials. A preliminary analysis was performed on the data for those subjects who were instructed not to move their eyes during a trial and those who received the same instructions, but whose eye movements were monitored and trials skipped when their eyes were found to move away from the central fixation. For the group whose eye movements were monitored, $9 \%$ of the trials were repeated due to eye movements, indicating that instructions not to move the eyes were generally followed.

Two separate ANOVAs of the RT data were performed in order to determine if there were significant differences in the results between the groups with and without eye movement recordings. One ANOVA was performed on a complete factorial design for 10 subjects who participated without eye movement recordings and for four subjects who participated with eye movement recordings. A second ANOVA was performed on a complete factorial design for four subjects who had participated in both procedures. The results showed that the trends in the data were virtually identical whether eye movements were recorded or not, as neither the between-subjects ANOVA nor the within-subjects ANOVA found the eye movement recording variable to interact with any of the other factors. The only significant effect found for eye movement recording was that the four subjects who participated only in the eye movement recording group were significantly faster in responding overall than the 10 subjects whose eye movements were not recorded $[F(12,144)=3.5, p<.001]$. Since the recording variable did not have any significant interactions with any of the other factors, the two groups were combined into a single group. This was accomplished for the four participants who participated in both groups by pooling the data and averaging to find a single mean score for each subject in all cells of the design.

\section{RT Data}

The mean RTs for the four cue conditions plotted against the frame containing the target (2-16) are shown in Figure 2. The RT data were analyzed using a 2 (L vs. $\mathrm{R}$ target) $\times 4$ (cue condition) $\times 5$ (frame) ANOVA. The five levels of the frame factor were achieved by collapsing the data from the 15 experimental frames that could contain the target into sets of three - that is, Frames 2-4, 5-7, 8-10, 11-13, and 14-16 (see Table 1).

The results revealed significant effects for all three factors. Mean RT differed across conditions, with the longest RT found in the control condition $(593 \mathrm{msec})$, followed by the temporal and spatial cue conditions (561 msec and $559 \mathrm{msec}$, respectively), and the shortest RT was obtained in the spatiotemporal cue condition (515 $\mathrm{msec})$ $[F(3,51)=17.1, p<.001]$. Mean RT was shorter for R tar- 


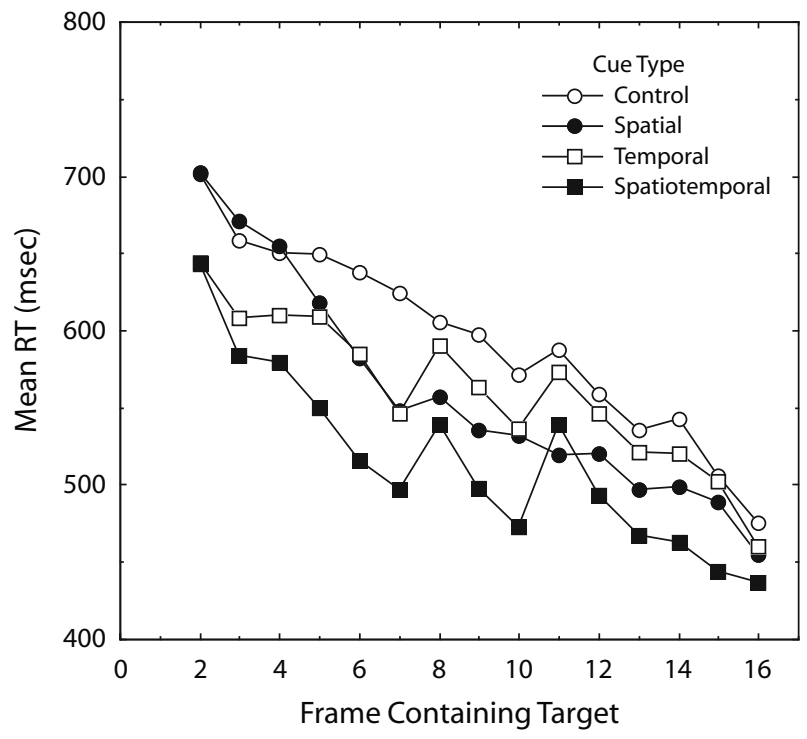

Figure 2. Mean RT plotted against target frame (2-16) in the RSVP sequence for the four cuing conditions.

gets $(532 \mathrm{msec})$ than for L targets $(579 \mathrm{msec})[F(1,17)=$ $19.3, p<.001]$. Finally, mean RT was shown to decrease across frame number $[F(4,68)=83.9, p<.001]$.

The data showed two significant interactions. The R/L RT difference decreased smoothly across frame number from $63 \mathrm{msec}$ in the early frames to $24 \mathrm{msec}$ in the later frames $[F(4,68)=3.52, p=.011]$. There was also a significant interaction between frame and condition, with minimal spatial cue effects early in the RSVP sequence and minimal temporal cue effects later in the sequence $[F(12,204)=4.4$, $p<.001$ ] (see Figure 2 and Tables 1 and 2).

The mean RT data shown in the right-hand column of Table 1 were arranged into a $2 \times 2$ table, with presence or absence of spatial cues crossed against presence or absence of temporal cues. In this way, the overall benefit of temporal cues was found to be $38 \mathrm{msec}[F(1,17)=19.3, p<$ $.001]$, and the overall benefit of spatial cues was $40 \mathrm{msec}$ $[F(1,17)=28.3, p<.001]$. The benefit for spatiotemporal combination cues over the control, no-cue condition RTs was $78 \mathrm{msec}$, which was statistically equivalent to the additive combination of separate spatial and temporal benefits. That is, there was no significant interaction between the effects of spatial and temporal cues $(F<1)$ suggesting that the effects of spatial and temporal cuing were independent. In order to assess the power of the test for independence, the effect size for the interaction was first estimated, obtaining a value for Cohen's $d$ of 0.073 (i.e., the cell means differed at most by only a few msec from the values expected from the independence assumption). This is less than a "small" effect size of 0.20 , using Cohen's (1988) guidelines. When the observed estimates of error variance were used to determine the power of the interaction test to detect a small effect size of $d=0.20$ (using the methods suggested by Maxwell \& Delaney, 2004), the power would have been in excess of $0.8 .^{1}$

A separate ANOVA was performed on the cue-target lag data from the individual trial blocks in the temporal and spatiotemporal cue conditions. Both lag and condition had significant effects $[F(5,85)=22.7$ and $F(1,17)=25.6$, respectively, both $p \mathrm{~s}<.001]$. In general, the condition main effect replicated the finding in the previous ANOVA, and the lag effect indicated that RTs were generally shorter for longer cue-target lags. However, these two factors also entered into a significant interaction $[F(5,85)=5.5, p<$ .001]. Thus, mean RTs were significantly shorter in the spatiotemporal cue condition at lags two or longer than in the temporal condition (see Figures 3 and 4). The delayed advantage found for the spatial-temporal cue over the temporal cue alone condition was similar to that observed between the spatial cue and control conditions, in that the spatial cue effect was absent in the first few frames after the cue (see Figure 2).

Two aspects of the RT data indicated that some of the cuing effects might have been reduced artifactually due to some of the temporal constraints imposed on using the cues. Specifically, it can be observed in Figure 2 that spatial cues were relatively ineffective early in the RSVP sequence and temporal cues were relatively ineffective late. This interaction is captured by the slopes of the regression lines relating mean RT to target frame number, as shown in Table 2. Without undue speculation, the lack of spatial effects early in the sequence could be due to the time necessary to inspect the cue and reallocate attention to the letter location adjacent to the cue. It could well take several hundred msec before the spatial cue resulted in optimal allocation of attention. Similarly, temporal cues resulted in an advantage over the control condition results, but the cuing effect diminished later in the RSVP sequence. This reduction in cue effectiveness is likely due to the fact that the passage of time during control trials without the target appearing increases the likelihood that the target will soon appear. In other words, target probability in any frame has an increasing hazard function of the frame number by which it has not yet appeared. Thus, the passage of time during a trial before the target appears acts like a temporal

Table 1

Mean Response Times (RTs, in Milliseconds) and Percent Errors (\%E) for Each Cuing Condition Across Frame Triplets in the RSVP Sequence

\begin{tabular}{|c|c|c|c|c|c|c|c|c|c|c|c|c|}
\hline \multirow[b]{3}{*}{ Condition } & \multicolumn{10}{|c|}{ Frame Numbers } & & \\
\hline & \multicolumn{2}{|c|}{$2-4$} & \multicolumn{2}{|c|}{$5-7$} & \multicolumn{2}{|c|}{$8-10$} & \multicolumn{2}{|c|}{$11-13$} & \multicolumn{2}{|c|}{$14-16$} & \multicolumn{2}{|c|}{ Mean } \\
\hline & $\mathrm{RT}$ & $\% \mathrm{E}$ & $\overline{\mathrm{RT}}$ & $\% \mathrm{E}$ & $\mathrm{RT}$ & $\% \mathrm{E}$ & $\mathrm{RT}$ & $\% \mathrm{E}$ & $\overline{\mathrm{RT}}$ & $\% \mathrm{E}$ & RT & $\% \mathrm{~B}$ \\
\hline Control & 670 & 9 & 637 & 9 & 591 & 9 & 561 & 8 & 508 & 8 & 593 & 8.6 \\
\hline Spatial cues & 676 & 2 & 583 & 2 & 541 & 3 & 512 & 4 & 481 & 7 & 559 & 3.6 \\
\hline Temporal cues & 621 & 7 & 580 & 6 & 563 & 7 & 546 & 7 & 494 & 5 & 561 & 6.4 \\
\hline Spatiotemporal cues & 602 & 6 & 521 & 3 & 504 & 4 & 499 & 5 & 448 & 6 & 515 & 4.8 \\
\hline
\end{tabular}


Table 2

Linear Regression Parameters for the Lines of Best Fit for Response Time (RT) Plotted Against Target Frame Number (x) for the Four Cuing Conditions Shown in Figure 1

\begin{tabular}{lcc}
\hline \multicolumn{1}{c}{ Condition } & Regression Line & $R^{2}$ \\
\hline Control & RT $=716-13.7 x$ & .96 \\
Spatial cues & RT $=699-15.6 x$ & .92 \\
Temporal cues & RT $=652-10.2 x$ & .86 \\
Spatiotemporal cues & RT $=619-11.6 x$ & .81 \\
\hline
\end{tabular}

cue in itself, reducing the mean RT across frames in the control condition and consequently diminishing the effectiveness of late temporal cues.

For these reasons, it was decided to truncate the trial sequence to remove artifactual reductions of cue effects and compare the cuing effects when both spatial and temporal cues were at their maximum effectiveness. For the following analysis, the RT data from the central Frames 5-13 only were used (i.e., the data from trials with targets in Frames 2, 3, 4, 14, 15, and 16 were eliminated).

A second artifactual source of reductions in cue effectiveness is visible in the lag data from the temporal and spatiotemporal cue conditions (see Figures 3 and 4). In these data, the temporal and combined cues at lag zero produce much smaller cuing effects than at any other lag. Again, it is likely that when the temporal or spatiotemporal cue occurs in the RSVP sequence, its processing takes long enough that the cue cannot be used effectively when the target and cue occur on the same frame. When the cue preceded the target by one or more frames, however, both the temporal and spatiotemporal cues produced large reductions in mean RT. Therefore, we decided to further reduce the data by eliminating all trials in the zero lag condition for temporal and spatiotemporal cues in Frames 5-13.

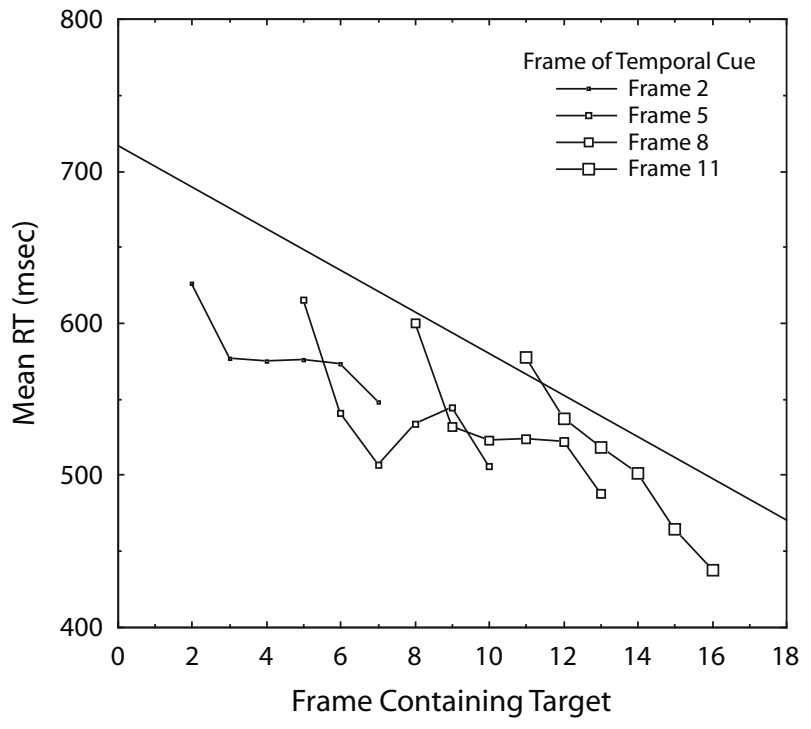

Figure 3. Mean RT plotted against target frame for temporal cues for each cue position (cues given on Frame 2, 5, 8, or 11 only). The straight line indicates the regression line for RT against frame for the control condition (see Table 2).
The reduced data were combined into a $2 \times 2$ table crossing the presence and absence of the two types of cues, and these data are shown in Table 3. As can be seen in the table, the effects of spatial and temporal cues are clearly additive (interaction, $F<1$ ). The effects of spatial cues were about $52 \mathrm{msec}$ overall, and the effects of temporal cues averaged about $64 \mathrm{msec}$. In both cases the effects of either cue were within $2 \mathrm{msec}$ of each other when the other cue was present or absent, supporting the statistical demonstration of their independence (see Table 3 ). ${ }^{2}$

\section{Error Data}

The error data (see Table 1) were analyzed using the same 2 (L vs. R target) $\times 4$ (cue condition) $\times 5$ (frame) ANOVA as was used for the overall RT data. Error rates differed across condition, with the smallest proportion of errors in the spatial condition (3\%), followed by the spatiotemporal and temporal cue conditions $(5 \%$ and $7 \%$, respectively), and the highest proportion of errors occurred in the control condition $(9 \%)[F(3,51)=32.4, p<.001]$. A significant interaction was found between frame and condition $[F(12,204)=4.5, p<.001]$. The error rates increased slightly across frame number in the spatial cue condition, whereas they were relatively flat across frame number in the other three conditions. Since both RT and error rates were highest in the control condition, it seems unlikely that the RT trends in the other conditions were compromised by speed-accuracy trade-offs. No other effects were significant in the error data.

\section{DISCUSSION}

The current results replicate and extend previous findings of benefits associated with the use of spatial and temporal cues in stimulus identification. Mean RTs for identifying which of two target letters appeared in an RSVP stream were reduced by almost equal amounts in response to spatial or temporal cues in comparison to a no-cue, control condition. The equivalent benefits for either type of cue can only be regarded as serendipitous, as the spatial cue reduced positional uncertainty from four possible spatial locations to one, and the temporal cue reduced sequential uncertainty from 15 possible frames to one. Nonetheless, the equivalently strong benefits observed for the two types of cues used in the present study enables a more powerful test of their presumed independence than if one or the other of them had been weak.

The benefits associated with spatial cues have been studied extensively (e.g., Posner, 1980). However, the importance and impact of temporal cues have only recently begun to be understood (e.g., Coull, Frith, Büchel, \& Nobre, 2000), and there are still questions about whether or not temporal attention can come under conscious control or if temporal attention is merely associated with some type of automatic motor preparation.

Coull et al. (2000) argued that temporal attention cues produce more than motor preparation. They found that orienting attention to a specific time interval engages processes other than those required for preparing a motor response. Their fMRI results showed that there was no anatomical 


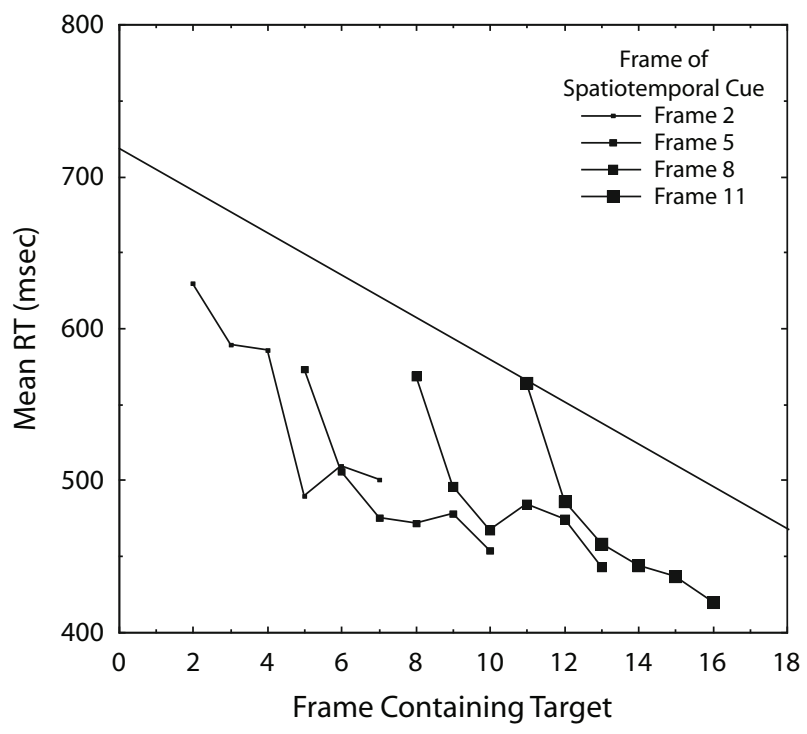

Figure 4. Mean RT plotted against target frame for spatiotemporal cues for each cue position (cues given on Frames 2, 5, 8, or 11 only). The straight line indicates the regression line for the control condition (see Table 2).

overlap between areas sensitive to motor preparation or timing (e.g., the medial premotor cortex, left putamen, and the bilateral thalamus) and areas sensitive to attentional aspects of temporal orienting. Instead, areas associated with attentional shifts, such as the left parietal cortex, were activated during the temporal orienting of attention.

Further arguments against the idea that temporal cues affect only motor response preparation come from behavioral studies. Correa et al. (2004) pointed out that if temporal cue effects are measured in a detection task, they cannot be used to discriminate cue effects on attention and perceptual processing from those affecting motor preparation. The present study's results using a discrimination task extend those of Coull et al.'s (2000) target detection task. Both studies showed temporal cuing effects, which lend support to the idea that the orienting of attention in time benefits selected stimulus processing and leads to temporal cue effects beyond simple motor preparation. These conclusions are supported by a recent study by Correa, Lupiáñez, and Tudela (2005). They showed that valid temporal cues for the location of a target in an RSVP stream facilitated sensitivity $\left(d^{\prime}\right)$ of the target discrimination response; a result incompatible with the assumption that temporal cues promote merely motor programming.

Correa et al. (2004) also argued that temporal orienting effects for both early and late expectancies are important for determining whether the underlying mechanisms are endogenous or voluntary. That is, if effects are found only for late expectancy, they could be determined by changes in alertness over time. In the present study, temporal cuing effects were found for both early expectancies (as early as lag 0 ) and also for late expectancies (as long as lag 5), which corresponds to about $520 \mathrm{msec}$ between the offset of the cue and onset of the target. Although temporal cues were usually less effective for the immediate frame (lag 0), they were about equally strong in producing benefits for lags 2 through 5 (see Figures 3 and 4). Thus, the present results provide evidence that temporal attention can indeed come under conscious control and be as beneficial as spatial attention in some task situations.

Temporal expectancy can also be used to explain the current findings of reductions in RTs across frame number. The idea is that, in single-target studies, the flow of time itself contains information about the upcoming likelihood of target occurrence. The more time that has elapsed before a target has appeared in the RSVP sequence increases the probability that it will occur soon (i.e., the hazard function steadily increases across frame number, since each frame is equally likely a priori to include the target). Niemi and Näätänen (1981) pointed out that if a target does not occur at an expected time, such as early in the trial, then expectancy might gradually peak later in the trial and consequently shorten RTs. The passage of time without a target occurring in effect then acts like a temporal cue, explaining the reduction in temporal cuing effects relative to the control condition as frame number increased in the present study. It should be pointed out however, that any increase in expectancy that might accrue during the RSVP stream acts as a general temporal cue for target occurrence and is not as effective as the specific temporal cues used here, even very late in the trial sequence (see Figure 3).

The present results also replicate and extend the work of Coull and Nobre (1998) by showing that there are independent effects of spatial and temporal cues on target detection and discrimination in a visual search task. The additive effects of temporal and spatial cues found in the present study are consistent with the idea that there are separate attentional mechanisms that direct attention to locations in space versus those used to allocate attention to specific events in time.

Although additivity of cuing effects in behavioral measures is consistent with the assumption of independence, it is not conclusive by itself. The additivity assumption is bolstered, however, by the results of recent neuroimaging research showing that temporal and spatial attention are reflected by activity in different brain areas (Griffin et al., 2001). Although an overlap of activation in the frontal and parietal regions was found between spatial and temporal orienting tasks, hemispheric lateralization was found within the common frontal-parietal system. Spatial attention was associated with activation primarily in the

Table 3

Mean Response Times (RTs, in Milliseconds) for the Four Cuing Conditions for Target Frames 5-13

\begin{tabular}{lrrc}
\hline & \multicolumn{2}{c}{ Spatial Cue } & \\
\cline { 2 - 3 } Pemporal Cue & No & Yes & Difference \\
\cline { 2 - 3 } Presented & 596 & 545 & 51 \\
No & 532 & 479 & 53 \\
Yes & 64 & 66 & \\
Difference & &
\end{tabular}

Note- The data for cue-target lag 0 have been removed from the temporal and spatiotemporal cuing conditions. The data show the additive combination of spatial and temporal cuing effects. 
right posterior parietal cortex. In contrast, temporal attention was associated with activation of the left parietal and inferior premotor cortex. Thus, different regions of the frontal-parietal system were activated depending on the type of information used to deploy attention.

Recently, Doherty et al. (2005) challenged the conclusion that spatial and temporal expectancies operate independently in object perception. They studied the perception of a moving circle that disappeared behind an occluding barrier and reappeared on the other side. The task was to respond if the circle had a black spot in it when it reappeared and to withhold a response of it did not. The main manipulation was the type of path that the circle followed before it disappeared behind the barrier. In one condition, its movement was regular over space and time, following an apparently linear path that resulted in high level of anticipation about when and where it should reappear after it passed behind the barrier. In other conditions, either its trajectory, or its apparent speed of motion were subjected to random perturbations to reduce the correct anticipation of the time, place, or both time and place of its reappearance. The results showed that RTs to detect the dot were shorter in both the temporally and spatially regular motion conditions. A further RT advantage was found when motion over both time and space were regular, resulting in additive effects of spatial and temporal expectancy, as in the present study.

In contrast to the RT data, Doherty et al.'s (2005) analysis of the evoked potentials recorded during dot-detection responses yielded a different pattern of results. Earlier literature had suggested that spatial expectation should enhance early visual components such as $\mathrm{P} 1$ and N1, as well as later components such as N2 and P3. Temporal expectation, on the other hand should not modulate P1 and $\mathrm{N} 1$, but have an effect only on the latter components (Griffin et al., 2002; Miniussi, Wilding, Coull, \& Nobre, 1999). Doherty et al. reported the expected results that spatial expectation enhanced P1 amplitude, whereas temporal expectancy had no effect. However, in contrast with both earlier results and their own behavioral data, temporal and spatial expectancy interacted to produce a larger P1 amplitude than expected on the basis of their independent effects alone. No other components analyzed (i.e., N1, N2, and P3) showed any interactive effects of the two types of expectancies. Doherty et al. concluded that "spatial and temporal expectation acting alone optimized the neural processing of target stimuli through distinct mechanisms. However, when both expectations were present together, they cooperated to enhance further the perceptual analysis of relevant stimuli" (p. 8264).

Despite this claim, there is no evidence in their RT data to support a superadditive interaction (in fact, the nonsignificant interaction they report in the RT data is on the subadditive side). Further, the majority of their ERP measurements show no spatial-temporal interactions. We are left with the impression that there is something about the stimuli they used, something special about tracking an object in apparent motion, that enhances P1, whereas deviations in velocity or position destroy much of the normal object integration processes reflected in $\mathrm{P} 1$. The enhanced integration of object features might have little to do with selection of an irrelevant feature (the addition of a target dot), thus it is possible that $\mathrm{P} 1$ did not reflect temporal selection that influenced the behavioral measure in Doherty et al.'s study as much as integration of spatial and temporal information useful for tracking a moving object. Obviously, further research is necessary to sort out the relative consistencies and inconsistencies between behavioral and neuroimaging results in the attention literature.

In summary, the present study replicated and extended most previous findings of spatial and temporal benefits for attentional deployment in response to valid cues. Furthermore, the temporal cue effects were found at both early and late expectancies in a target discrimination task, which lends support to the idea that temporal orienting of attention is under voluntary control and is more than mere motor preparation. Sperling and Weichselgartner (1987) compared temporal attention to a gate that opens to allow information to be processed and then closes so that other information is inhibited from further processing. The gate analogy was also used by Tse, Intriligator, Rivest, and Cavanagh (2004), who added the claim that attention enhances the temporal resolution of visual processing such that an attended episode seems to occur in slow motion or to pop out from other episodes. Therefore, it is possible that temporal attention not only improves motor preparation but also acts like a gate to select relevant information for processing while inhibiting distractions from irrelevant preceding and following information.

The additive effects found in the present study when spatial and temporal cues were combined supports, but does not confirm, the hypothesis that spatial and temporal deployment of attention rely on different underlying neural mechanisms. Neuroimaging studies have shown that spatial and temporal selective attention generally rely on different areas of the brain and occur at different times in stimulus processing (Griffin et al., 2001, 2002; but see also Doherty et al., 2005). Spatial attention has been associated with activation in the right posterior parietal cortex during early stimulus processing whereas temporal attention has been associated with activation in the left parietal cortex and the inferior premotor cortex during later stimulus processing. Therefore, the present results provide converging evidence in support of most neuroimaging studies indicating that at least early visual processes involved in selective attention to locations in space and episodes in time are independent. The present results do not preclude the notion that, at later stages of visual processing after attentional selection is complete, object and event information are combined in order to guide understanding of dynamic interactions among objects over time.

\section{AUTHOR NOTE}

We acknowledge support from a grant from the General Research Fund of the University of Kansas. Much of the research reported here was included in a master's thesis submitted to the University of Kansas by the first author. Portions of this research were reported at the Annual Meeting of the Psychonomic Society in Vancouver, Canada, 2003. We thank Devin Land for his assistance in setting up the eye movement recording apparatus; Brian Wilson, Satha Phongsatha, and Ming-Chou Ho for assistance with programming; and Jon Templin for assistance with statistical analyses. Correspondence concerning this article should be addressed to 
A. MacKay, Department of Psychology, University of Kansas, 1415 Jayhawk Boulevard, Lawrence, KS 66045 (e-mail: anm1619@ku.edu).

\section{REFERENCES}

CASINI, L., \& MACAR, F. (1997). Effects of attention manipulation on judgments of duration and of intensity in the visual modality. Memory \& Cognition, 25, 812-818.

CoHEN, J. (1988). Statistical power analysis for the behavioral sciences (2nd ed.). Hillsdale, NJ: Erlbaum.

Correa, A., Lupiáñez, J., Milliken, B., \& Tudela, P. (2004). Endogenous temporal orienting of attention in detection and discrimination tasks. Perception \& Psychophysics, 66, 264-278.

Correa, A., Lupiáñez, J., \& Tudela, P. (2005). Attentional preparation based on temporal expectancy modulates processing at the perceptual level. Psychonomic Bulletin \& Review, 12, 328-334.

Coull, J., Frith, C., Büchel, C., \& Nobre, A. (2000). Orienting attention in time: Behavioural and neuroanatomical distinction between exogenous and endogenous shifts. Neuropsychologia, 38, 808-819.

Coull, J., \& Nobre, A. (1998). Where and when to pay attention: The neural systems for directing attention to spatial locations and to time intervals as revealed by both PET and fMRI. Journal of Neuroscience, 18, 7426-7435.

Doherty, J. R., Rao, A., Mesulan, M. M., \& Nobre, A. C. (2005). Synergistic effect of combined temporal and spatial expectations on visual attention. Journal of Neuroscience, 25, 8259-8266.

Griffin, I., Miniussi, C., \& Nobre, A. (2001). Orienting attention in time. Frontiers in Bioscience, 6, 660-671.

Griffin, I., Miniussi, C., \& Nobre, A. (2002). Multiple mechanisms of selective attention: Differential modulation of stimulus processing by attention to space or time. Neuropsychologia, 40, 2325-2340.

JiANG, Y., \& ChUN, M. (2001). The influence of temporal selection on spatial selection and distractor interference: An attentional blink study. Journal of Experimental Psychology: Human Perception \& Performance, 27, 664-679.

Juola, J. F., Bouwhuis, D. G., Cooper, E. E., \& Warner, C. B. (1991). Control of attention around the fovea. Journal of Experimental Psychology: Human Perception \& Performance, 17, 125-141.

KarLin, L. (1959). Reaction time as a function of foreperiod duration and variability. Journal of Experimental Psychology, 58, 185-191.

Kristuánsson, Á., \& NaKaYAma, K. (2002). The attentional blink in space and time. Vision Research, 42, 2039-2050.

MACKWORTH, N. (1948). The breakdown of vigilance during prolonged visual search. Quarterly Journal of Experimental Psychology, 1, 6-21.

Maxwell, S. E., \& Delaney, H. D. (2004). Designing experiments and analyzing data: A model comparison perspective (2nd ed.). Mahwah, NJ: Erlbaum.

Milliken, B., Lupiáñez, J., Roberts, M., \& Stevanovski, B. (2003).
Orienting in space and time: Joint contributions to exogenous spatial cuing effects. Psychonomic Bulletin \& Review, 10, 877-883.

Miniussi, C., Wilding, E., Coull, J., \& Nobre, A. (1999). Orienting attention in time: Modulation of brain potentials. Brain, 122, 1507-1518.

Mishkin, M., Ungerleider, L., \& Macko, K. A. (1983). Object vision and spatial vision: Two cortical pathways. Trends in Neurosciences, 6, 414-417.

Nakayama, K., \& Mackeben, M. (1989). Sustained and transient components of visual attention. Vision Research, 29, 1631-1647.

NIEMI, P., \& NäÄTÄNEN, R. (1981). Foreperiod and simple reaction time. Psychological Bulletin, 89, 133-162.

PosNer, M. (1980). Orienting of attention. Quarterly Journal of Experimental Psychology, 32, 3-25.

RAYMOND, J., ShaPIRo, K., \& ARNELl, K. (1992). Temporary suppression of visual processing in an RSVP task: An attentional blink? Journal of Experimental Psychology: Human Perception \& Performance, 18, 849-860.

Schneider, W., \& Zuccolotto, A. (2002). E-Prime user's guide. Pittsburgh, PA: Psychology Software Tools.

SPerling, G., \& WeichSElgartner, E. (1987). Dynamics of automatic and controlled visual attention. Science, 238, 778-780.

Tse, P., Intriligator, J., Rivest, J., \& Cavanagh, P. (2004). Attention and the subjective expansion of time. Perception \& Psychophysics, 66, 1171-1189.

\section{NOTES}

1. In a further test of the independence assumption, the correlations between the RTs across subjects for the two temporal cue conditions (i.e., presence or absence of the temporal cue) were found for the cases in which the spatial cue was absent and when it was present. These were .756 and .778 , respectively (both $p$ s $<.001$ ). The correlations between the RTs across subjects for the two spatial cue conditions (i.e., presence or absence of the spatial cue) were .800 and .801 , respectively, for the temporal cue absent and present conditions (both $p$ s $<.001$ ). Note that if the mean RTs for either cue condition were unaffected by the presence or absence of the other cue, the respective pairs of correlations would have been identical, which for all practical purposes is what we observed.

2. As an additional test of the independence of spatial and temporal cue effects for the data shown in Table 3, the differences were found between the RTs in the temporal cue condition and those in the spatiotemporal cue condition at each temporal cue lag. The additional benefits for the spatial cues were found to be $41,49,63,59$, and $48 \mathrm{msec}$ for the lag 1 to lag 5 conditions, respectively. The largest difference between these values (i.e., $22 \mathrm{msec}$ for lag $1 \mathrm{vs}$. lag 3 ) was not significant by a $t$ test.

(Manuscript received February 7, 2006; revision accepted for publication February 2, 2007.) 